\title{
The Impact of Bariatric Surgery on the Muscle Mass in Patients with Obesity: 2-Year Follow-up
}

\author{
Marta Comas Martínez ${ }^{1,2} \cdot$ Enzamaria Fidilio Meli ${ }^{1} \cdot$ Fiorella Palmas Candia ${ }^{1} \cdot$ Francesca Filippi $^{3} \cdot$ Ramon Vilallonga $^{4}$. \\ Efrain Cordero ${ }^{1} \cdot$ Irene Hernández ${ }^{1}$. Alba Zabalegui Eguinoa ${ }^{1} \cdot$ Rosa Burgos $^{1} \cdot$ Anna Vila $^{2} \cdot$ Rafael Simó $^{1,4,5}$. \\ Andreea Ciudin ${ }^{1,4,5}$
}

Received: 9 September 2021 / Revised: 11 November 2021 / Accepted: 14 November 2021 / Published online: 30 November 2021

(c) The Author(s) 2021

\begin{abstract}
Purpose Bariatric surgery (BS) induces a significant and sustained weight loss in patients with severe obesity (SO). Nevertheless, apart from significantly reducing body fat, fat-free mass (FFM) might also be lost. At present, there is little and controversial data in the literature regarding the impact of BS on FFM. In recent years, bioimpedance (BIA) has emerged as a reliable test to assess body composition easily to use in the daily clinical practice. On the bases, the aim of the present study is to evaluate the impact of BS on the FFM, evaluated by means of BIA.

Material and Methods This is a prospective, observational study, including consecutive patients with SO that underwent BS between February 2018 and February 2019 at our center. At baseline, 1, 6, 12, and 24 months after the BS, all the patients underwent complete medical history, physical and anthropometric evaluation, and body composition assessment by means of BIA (using Bodystat QuadScan4000®).

Results Eighty-five patients with SO were recruited, $72.9 \%$ females, aged $45.54 \pm 9.98$ years, pre-BS BMI $43.87 \pm 6.52 \mathrm{~kg} /$ $\mathrm{m}^{2}$. FFM significantly decreased continuously after BS at all timepoints. The loss of FFM 24 months post-BS accounted for approximately $21.71 \pm 13.9 \%$ of the total weight loss, and was independent of BS technique or protein metabolism. Pre-BS HOMA-IR and FFM were independent predictors of FFM at 24 months.

Conclusions Significant and early loss of FFM in patients with SO that undergo BS was seen, not related to protein metabolism parameters or the BS technique used, suggesting an independent mechanism.
\end{abstract}

Keywords Morbid obesity $\cdot$ Fat-free mass, Bariatric surgery $\cdot$ Sarcopenic obesity

\section{Introduction}

Key Points • Significant muscle loss occurs starting early after bariatric surgery.

- The muscle loss is independent on the bariatric surgery technique.

- The muscle mass is related to resting energy expenditure

Rafael Simó

rafael.simo@ vhir.org

$\triangle$ Andreea Ciudin

aciudin@vhebron.net

1 Endocrinology and Nutrition Department, Hospital Universitari Vall d'Hebron, Diabetes and Metabolism Research Unit, Vall d'Hebron Institut de Recerca (VHIR), Universitat Autònoma de Barcelona, Pg Vall d'Hebron 119-129, 08035 Barcelona, Spain

2 Research Group M3O, Methodology, Methods, Models and Outcomes of Health and Social Sciences, Faculty
The prevalence of obesity has increased worldwide over the last 50 years, reaching pandemic levels, in particular severe obesity (SO). Obesity represents a significant public health

of Health Sciences and Welfare, University of Vic - Central University of Catalonia, Barcelona, Spain

3 Clinical Pharmacology Department, University Hospital Vall d'Hebron, Barcelona, Spain

4 Endocrine, Metabolic and Bariatric Unit, General Surgery Department, Hospital Universitari Vall d'Hebron, Universitat Autònoma de Barcelona, Barcelona, Spain

5 Centro de Investigación Biomédica en Red de Diabetes y Enfermedades Metabólicas Asociadas (CIBERDEM), Instituto de Salud Carlos III (ISCIII), Madrid, Spain 
challenge, and it is associated with a significant economic burden on the health systems of developed countries, mainly due to the associated comorbidities, in particular type 2 diabetes (T2D) [1-3]. Additionally, the loss of muscle mass and/or function, also known as sarcopenia, was related to metabolic disorders, like T2D, aging, and poor quality of life. $[4,5]$. Sarcopenic obesity (OS) is the combination of low muscle mass and strength with increased fat mass, and it has been associated with adverse health outcomes [6]. A recent meta-analysis found that the presence of OS was associated with a higher risk (OR 1.38, 95\% CI [1.27-1.50]) of T2D than with each condition separately (obesity or sarcopenia) [7]. The complete underlying mechanism is still unclear, but it seems that there is a bi-directional relationship, having as main factors chronic inflammation and insulin resistance (IR).

Physiologically, the maximum level of skeletal muscle mass and strength is reached between 30 and 50 years of age, and starting from this point, the muscle mass is decreasing as part of the aging process [8]. Nevertheless, several conditions can alter the physiological evolution of the muscle mass at earlier ages, such as associated obesity, rapid weight loss after diet, and physical inactivity.

The use of rapid weight-loss diets, such as very-lowcalorie diet (VLCD), has been shown to have a significant weight loss effect and a significant impact on muscle mass reduction. The impact on muscle mass has been seen to be greater than the impact on fat mass [9].

Different methods are available to assess muscle mass and body composition such as bioimpedance analysis (BIA), dual-energy X-ray absorptiometry (DXA), computed tomography $(\mathrm{CT})$, and magnetic resonance imaging (MRI). DXA is still considered the gold standard method in clinical practice and investigation - and used the diagnosis criteria EWGSOP2 for sarcopenia [10]. However, DXA is expensive, needs particular space, has subject-related limitations (maximum weight $160 \mathrm{~kg}$ ), and provides only quantitative skeletal muscle mass evaluation. On the other hand, BIA is a relatively simple, quick, and non-expensive method for assessing body composition [11-13] and provides data on the muscle quantity and estimated quality.

Bariatric surgery (BS) is at present the most successful treatment for SO, in terms of significant and sustainable weight loss, leading to an important improvement in obesityrelated comorbidities and quality of life [14]. At present, there is little and controversial data regarding the relationship between bariatric surgery and skeletal muscle mass. Furthermore, the data and the methods that were used in the different studies are heterogeneous (some used muscle mass estimation based on mathematical equations, while others use more direct methods) $[15,16]$.
On these bases, the aims of the present study were (a) to evaluate the impact of BS on FFM in patients with MO and (b) to explore biomarkers for FFM evolution after the BS.

\section{Material and Methods}

We performed a prospective, observational study, including patients with SO, consecutively attended at the Morbid Obesity Unit of our centre, that underwent BS between February 2018 and February 2019. The present study, part of the PREDIBAR trial (NCT 03784508), was approved by the local Ethics Committee (PR(AG)320/2018) and carried out in accordance with the Declaration of Helsinki. All the patients signed the informed consent form before the inclusion in the study.

Inclusion criteria are as follows: (a) age between 18 and 60 years, (b) SO fulfilling criteria for BS according to our protocol (BMI $>40 \mathrm{~kg} / \mathrm{m}^{2}$ regardless of the comorbidities or BMI $>35 \mathrm{~kg} / \mathrm{m}^{2}$ with at least 1 comorbidity related to obesity, (c) previous accomplishment of the preoperative protocol for BS at our site, (d) written informed consent form.

Exclusion criteria are as follows: (a) patients undergoing evaluation for a second-step surgery; (b) unable to perform the post-BS follow-up at our site at least during 2 years; (c) subjects unable perform BIA (e.g. limb amputation, unwilling, and unable to fast for more than 8 hours); (d) presence of other conditions that can affect the muscle mass as per investigator criteria (e.g. immobilization, myopathies, and endocrinopathy such as Cushing disease); (e) severe concomitant pathology (cardiovascular, cerebrovascular, pulmonary, renal, or neoplastic) that can limit the participation in the study as per investigator criteria; (f) the use of drugs that can affect the muscle mass (e.g. corticosteroids); (g) active abuse of drugs or alcohol; (h) uncontrolled psychiatric illness or eating disorders.

All patients underwent baseline, at 1, 6, 12, and 24 months after the BS performance: complete medical history, physical, anthropometric, as per preoperative protocol for BS at our site. Additionally, biochemical analysis was performed including $\mathrm{HbA} 1 \mathrm{c}$, insulin levels, homeostatic model assessment for insulin resistance (HOMA-IR), and sensitive parameters from protein metabolism (transthyretin).

- HOMA-IR was calculated using the formula: [Fasting glucose $(\mathrm{mmoL} / \mathrm{L}) *$ fasting insulin $(\mu \mathrm{UI} / \mathrm{mL})] / 22.5$

- BMI was calculated by the formula: weight $(\mathrm{kg}) / \mathrm{height}$ $\left(\mathrm{m}^{2}\right)$

- \%Excess of weight loss (\%EWL) was calculated by the formula: (W $(\mathrm{kg})$ initial - W $(\mathrm{kg})$ final $) /(\mathrm{W}(\mathrm{kg})$ initial $-\mathrm{W}(\mathrm{kg})$ ideal $) * 100$ 
- \%Total weight loss (\%TWL) was calculated by the formula: (W $(\mathrm{kg})$ initial - W (kg) final)/(W (kg) initial) * 100

Body muscle mass was evaluated by means of BIA [17]. The BIA device used in our study was Bodystat QuadScan $4000 \AA$, a multi-frequency device. The measurement is performed by placing two electrodes on the wrist and hand and two electrodes on the foot and ankle on the same side of the body. BIA can be carried out both in the outpatient clinic and in the hospitalization floors since these devices can be transported easily [18]. The patients included in the study were asked to fulfil the standardized conditions to perform a BIA: avoid physical exercise the previous $8 \mathrm{~h}$; fasting 6-8 $\mathrm{h}$ before the measurement, including water; have previously removed the hair from the limbs and all metal objects that may interfere with the measurement; if the patient wears a prosthesis or implant, the measurement is made on the opposite side [19].

The variables that were collected from the BIA evaluation were fat mass $(\mathrm{FM})(\mathrm{kg})$, fat-free mass $(\mathrm{FFM})(\mathrm{kg})$, fat-free mass index (FFMI) $\left(\mathrm{kg} / \mathrm{m}^{2}\right)$, and phase angle (PA) $\left(^{\circ}\right)$. The PA is considered an indicator of cellular integrity; it allows the interpretation of the capacity of the muscle cell to transmit the electrical stimulus produced by the BIA apparatus. Low PA values have been correlated with a worse prognosis and higher comorbidities [17] [20].

Statistical Analysis BM SPSS statistical software version 24 was used. Continuous variables are expressed as means \pm standard deviation (SD) for normal distributed variables and median \pm interquartile range (IQR) for non-normal distributed variables. Categorical variables are expressed with percentages. For differences between groups in continuous variables ANOVA, Student's $t$ test or Mann-Whitney $U$ test was used while $\chi^{2}$ was used for categorical variables. Spearman correlation and regression logistics analysis were used to explore the relation between different variables. A $p$-value $<0.05$ was considered statistically significant.

\section{Results}

A total of 90 consecutive patients with SO undergoing preoperative evaluation for BS at our site were recruited. Eighty-five patients underwent BS and had at least 24 months of follow-up at our site and were included in the study. The baseline characteristics of the patients are shown in Table 1.

Patients with T2D were treated with diet (100\%), metformin (90\%), aGLP-1 (62.4\%), iSGLT-2 (13.2\%), iDPP4 $(45.1 \%)$, and insulin (2.3\%). For the calculation of HOMAIR, the patients treated with insulin were excluded. The
Table 1 The baseline characteristics of the patients included in the study

\begin{tabular}{ll}
\hline$N$ & 85 \\
\hline Gender (females \%) & $62(72.9 \%)$ \\
Age (years) mean \pm SD & $45.54 \pm 3.38$ \\
Pre-BS BMI $\left(\mathrm{kg} / \mathrm{m}^{2}\right)$ mean $\pm \mathrm{SD}$ & $43.87 \pm 6.52$ \\
Roux-en-Y gastric bypass (RYGB) (\%) & $48(57 \%)$ \\
Sleeve gastrectomy (SG) (\%) & $36(43 \%)$ \\
HbA1c (\% DCCT) mean $\pm \mathrm{SD}$ & $5.89 \pm 0.96$ \\
HOMA-IR mean $\pm \mathrm{SD}$ & $6.13 \pm 4.26$ \\
Type 2 diabetes $(\%)$ & $40(47 \%)$ \\
Arterial hypertension $(\%)$ & $37(43.5 \%)$ \\
Hypercholesterolemia $(\%)$ & $22(25.9 \%)$ \\
Sleep apnea (\%) & $50(58.8 \%)$ \\
\hline
\end{tabular}

$B M I$, body mass index; $B S$, bariatric surgery; HOMA-IR, homeostatic model assessment for insulin resistance; $H b A l c$ (\% DCCT), glycated haemoglobin (\% Diabetes Control and Complications Trial)

evolution of weight, body composition evaluated by BIA and biochemical analysis after BS, is shown in Table 2 . All the patients presented remission of T2D, according to ADA guidelines [21] after the BS and was maintained at 24 months.

The FFM significantly decreased 1 month after the BS and continued to drop at 24 months. It should be noted that starting from 1 month until the end of the follow-up, FFM loss represented $>20 \%$ of the TWL (Table 2). FM also significantly decreased starting from 1 until 12 months after the BS. Between months 12 and 24 after the BS, FM stabilized and presented a slight tendency to increase, although without reaching statistical significance, as reflected by Fig. 1.

Additionally, basal metabolism rate (BMR), measured by BIA, significantly decreased 1 month after the BS compared to baseline and continued to decrease during the follow-up (Table 2). A positive correlation was found between FFM and the BMR at all timepoints, respectively. By contrast, FM showed no relation with the BMR as reflected by Fig. 2 .

Regarding the two BS techniques that were used in the study (RYGB and SG), as expected, RYGB was associated with greater global EWL and TWL than SG. No significant differences were seen between the two techniques in terms of body composition and biochemical analysis (HOMA-IR and transthyretin), except for the PA that was significantly lower after the RYGB, as reflected by Table 2 . We found significantly lower levels of PA after the BS in all patients, when compared to data in the literature from subjects with normal weight, of similar age and gender [22] (Table 3).

A multiple regression analysis, including variables as age, HbA1c levels, baseline FFM, baseline FM, baseline transthyretin, and HOMA-IR, was performed. We found that HOMA-IR and baseline FFM were the only independent 


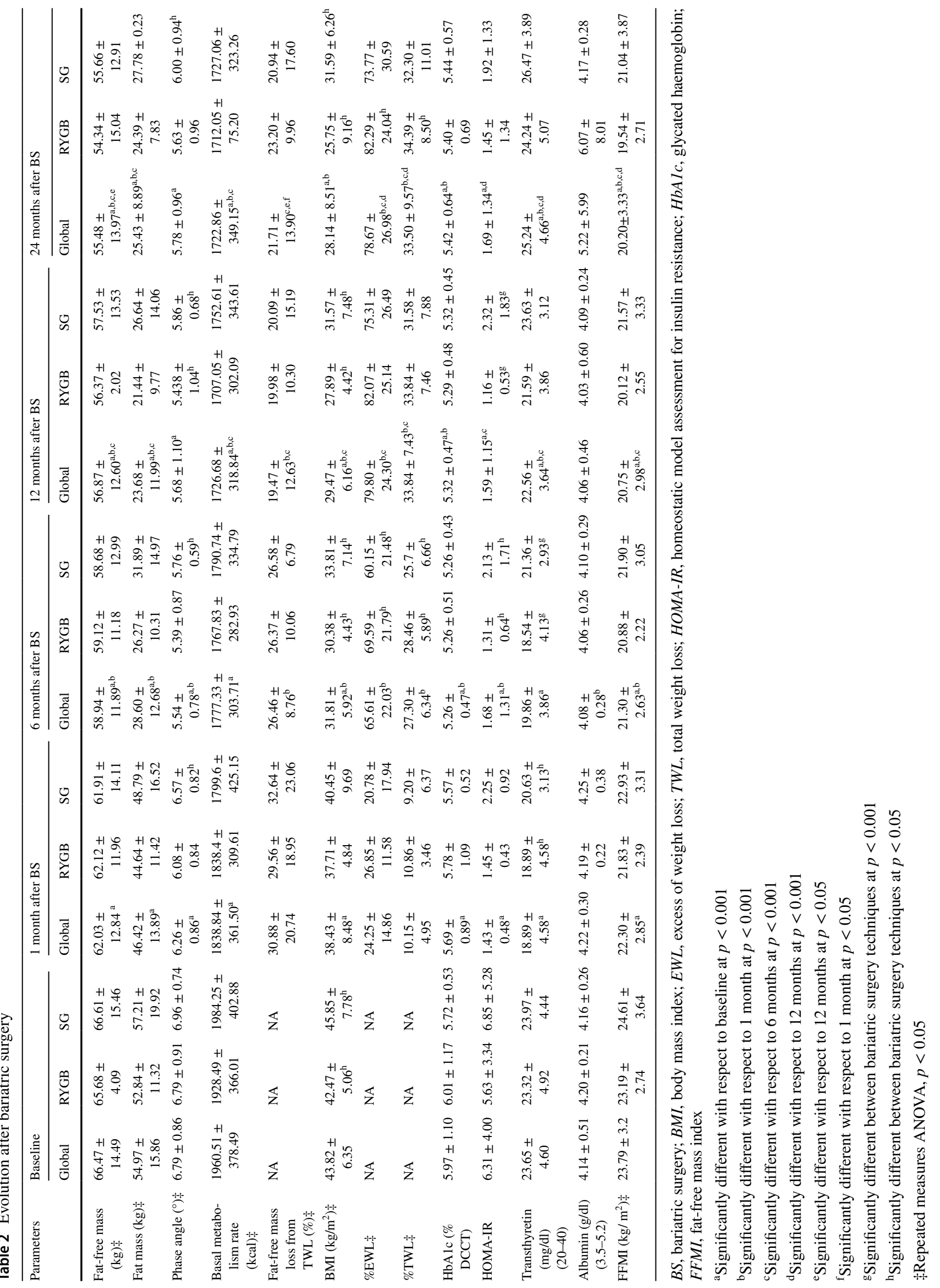



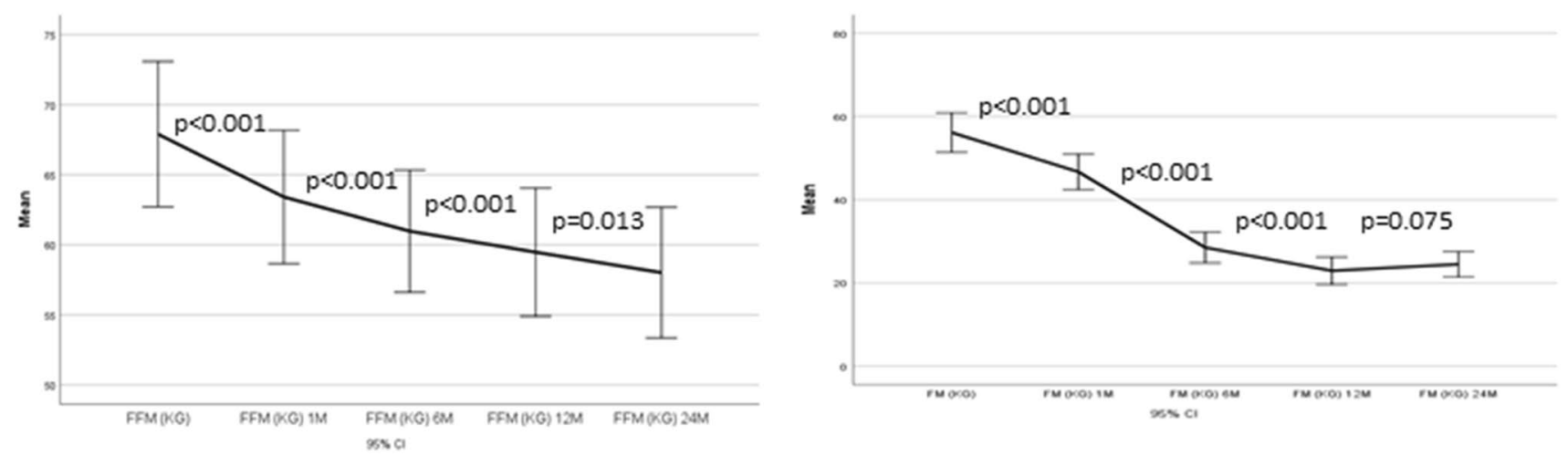

Fig. 1 Evolution of the FFM and FM during 24-month follow-up. FFM fat-free mass, FM fat mass, M months-reviewer 2
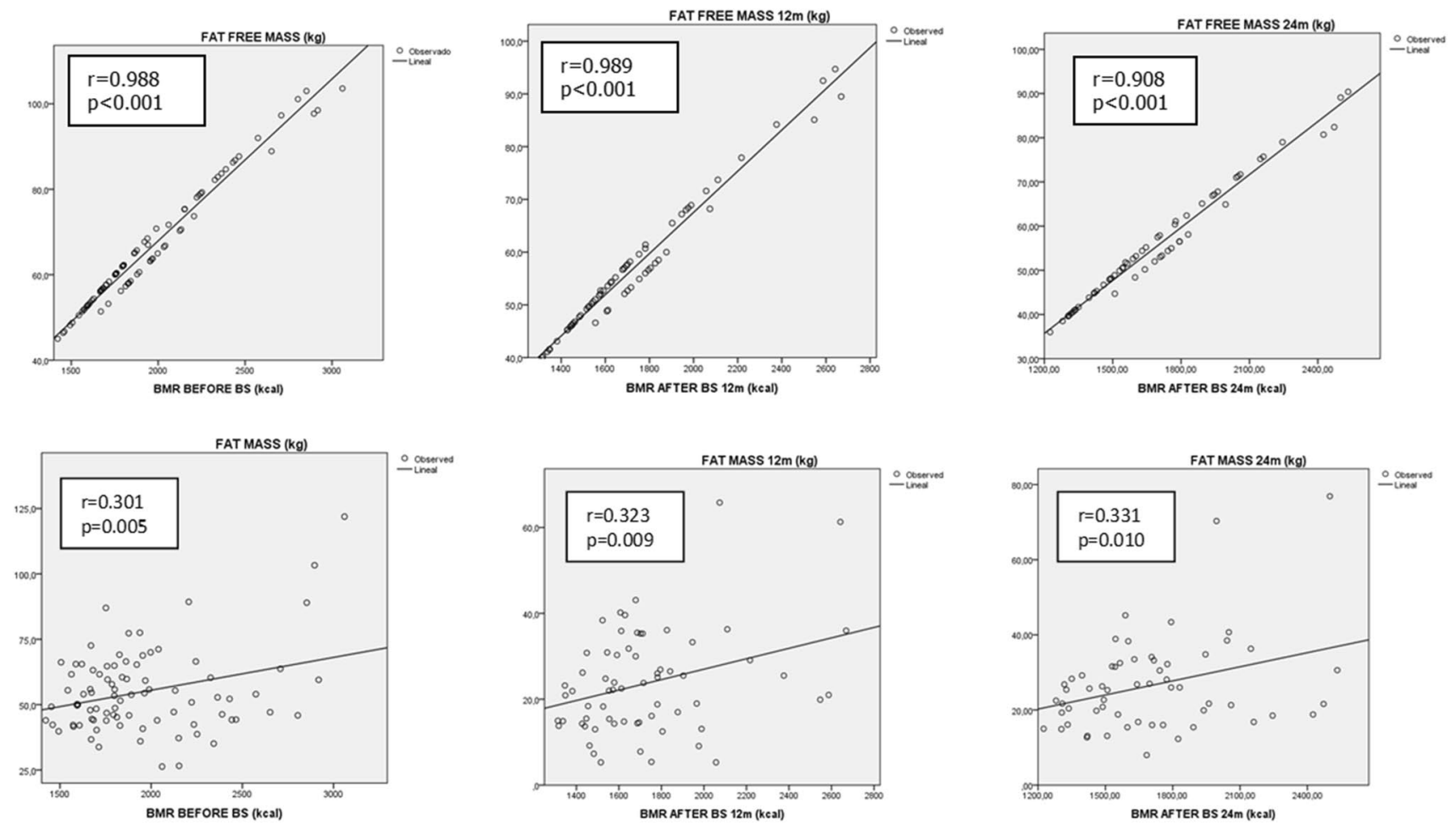

Fig. 2 Correlation between basal metabolism rate and body composition. BMR basal metabolism rate, BS bariatric surgery

predictors of FFM at 24 months after the BS as reflected by Table 4 .

\section{Discussion}

We showed in the present study that BS induces a significant and early loss of FFM after the BS, independent of protein metabolism, without significant differences between the BS techniques (RYGB and SG). In our study, BMI stabilized after 12 months post-BS. In exchange, body composition showed different profiles between 12 and 24 months: FFM continued to significantly drop while FM presented a tendency to increase, confirming the actual data in the literature [23-25] regarding the lack of precision of BMI to properly monitor the evolution after the BS.

Recently, our group showed that BS induces a significant reduction in basal metabolism rate starting 1 month after the BS in patients with extreme obesity that was associated with weight regain at 5-year follow-up [26]. It should be noted that in the study performed by Fidilio et al. [26], the BMR was measured by means of indirect calorimetry in patients with extreme obesity (BMI $>50 \mathrm{~kg} / \mathrm{m}^{2}$ ) and study of body composition was not performed. In the present study, we 
Table 3 The BS impact on the Phase angle levels in all patients

\begin{tabular}{|c|c|c|c|c|c|c|c|}
\hline & Baseline & 1 month & 6 month & 12 months & 24 months & Normal values [22] & $p \ddagger$ \\
\hline \multicolumn{8}{|l|}{ Females } \\
\hline $18-20$ years & $7.86 \pm 1.06$ & $6.46 \pm 0.3^{\mathrm{a}}$ & $6.25 \pm 0.59^{\mathrm{a}, \mathrm{b}}$ & $6.15 \pm 0.6^{\mathrm{a}, \mathrm{b}, \mathrm{c}}$ & $6.13 \pm 0.38^{\mathrm{a}, \mathrm{b}, \mathrm{c}, \mathrm{d}}$ & $7.04 \pm 0.85$ & 0.001 \\
\hline $21-29$ years & $7.4 \pm 0.74$ & $6.14 \pm 0.85^{\mathrm{a}}$ & $5.80 \pm 0.55^{\mathrm{a}, \mathrm{b}}$ & $5.9 \pm 0.76^{\mathrm{a}, \mathrm{b}, \mathrm{c}}$ & $5.68 \pm 0.53^{\mathrm{a}, \mathrm{b}, \mathrm{c}, \mathrm{d}}$ & $6.98 \pm 0.92$ & 0.0021 \\
\hline 30-39 years & $6.6 \pm 0.59$ & $5.96 \pm 0.76^{\mathrm{a}}$ & $5.39 \pm 0.66^{\mathrm{a}, \mathrm{b}}$ & $5.15 \pm 0.77^{\mathrm{a}, \mathrm{b}, \mathrm{c}}$ & $5.07 \pm 0.80^{\mathrm{a}, \mathrm{b}, \mathrm{c}, \mathrm{d}}$ & $6.91 \pm 0.85$ & $<0.001$ \\
\hline $40-49$ years & $6.12 \pm 0.9$ & $5.7 \pm 0.12^{\mathrm{a}}$ & $4.89 \pm 0.85^{\mathrm{a}, \mathrm{b}}$ & $4.85 \pm 1.04^{\mathrm{a}, \mathrm{b}, \mathrm{c}}$ & $5.07 \pm 0.16^{\mathrm{a}, \mathrm{b}, \mathrm{c}, \mathrm{d}}$ & $6.87 \pm 0.84$ & $<0.001$ \\
\hline \multicolumn{8}{|l|}{ Males } \\
\hline $18-20$ years & NA & NA & NA & NA & NA & $7.9 \pm 0.47$ & NA \\
\hline $21-29$ years & $7.7 \pm 0.3$ & $7.3 \pm 1.12^{\mathrm{a}}$ & $7.05 \pm 0.3^{\mathrm{a}, \mathrm{b}}$ & $7.15 \pm 0.4^{\mathrm{a}, \mathrm{b}, \mathrm{c}}$ & $6.35 \pm 0.91^{\mathrm{a}, \mathrm{b}, \mathrm{c}, \mathrm{d}}$ & $8.02 \pm 0.75$ & $<0.001$ \\
\hline 30-39 years & $7 \pm 0.56$ & $6.7 \pm 0.01^{\mathrm{a}}$ & $6.45 \pm 1.47^{\mathrm{a}, \mathrm{b}}$ & $6.25 \pm 0.75^{\mathrm{a}, \mathrm{b}, \mathrm{c}}$ & $6.14 \pm 0.54^{\mathrm{a}, \mathrm{b}, \mathrm{c}, \mathrm{d}}$ & $8.01 \pm 0.85$ & $<0.001$ \\
\hline $40-49$ years & $6.75 \pm 0.79$ & $6.85 \pm 0.46^{\mathrm{a}}$ & $5.63 \pm 0.68^{\mathrm{a}, \mathrm{b}}$ & $5.58 \pm 0.61^{\mathrm{a}, \mathrm{b}, \mathrm{c}}$ & $5.73 \pm 0.70^{\mathrm{a}, \mathrm{b}, \mathrm{c}, \mathrm{d}}$ & $7.76 \pm 0.85$ & $<0.001$ \\
\hline
\end{tabular}

${ }^{\text {a }}$ Significantly different respect to baseline at $p<0.001$; ${ }^{\mathrm{b}}$ significantly different with respect to 1 month at $p<0.001$; ${ }^{\mathrm{c}}$ significantly different with respect to 6 months at $p<0.001$; ${ }^{\mathrm{d}}$ significantly different with respect to 12 months at $p<0.001$; ;repeated measures ANOVA, $p<0.05$

Table 4 Independent parameters related to the FFM at 24 months after the BS

\begin{tabular}{|c|c|c|c|c|c|c|c|c|c|}
\hline \multicolumn{10}{|l|}{ Coefficients $^{\mathrm{a}}$} \\
\hline \multirow[t]{2}{*}{ Model } & \multicolumn{2}{|c|}{$\begin{array}{l}\text { Unstandardized coef- } \\
\text { ficients }\end{array}$} & \multirow{2}{*}{$\begin{array}{l}\text { Standardized } \\
\text { coefficients } \\
\text { Beta }\end{array}$} & \multirow[t]{2}{*}{$t$} & \multirow[t]{2}{*}{ Sig. } & \multicolumn{2}{|c|}{$95.0 \%$ confidence interval for B } & \multicolumn{2}{|c|}{ Collinearity statistics } \\
\hline & $B$ & Std. error & & & & Lower bound & Upper bound & Tolerance & VIF \\
\hline 1 (constant) & -8.750 & 8.300 & & -1.054 & .300 & -25.725 & 8.225 & & \\
\hline Age & .009 & .076 & .006 & .116 & .908 & -.147 & .164 & .884 & 1.131 \\
\hline FFM (kg) & .843 & .047 & .933 & 17.966 & .000 & .747 & .939 & .813 & 1.230 \\
\hline Transthyretin before & -.024 & .145 & -.008 & -.162 & .872 & -.320 & .273 & .812 & 1.231 \\
\hline HOMA-IR & .409 & .192 & .102 & 2.132 & .042 & .017 & .802 & .966 & 1.036 \\
\hline HbA1c levels & .800 & .610 & .065 & 1.313 & .200 & -.447 & 2.048 & .891 & 1.122 \\
\hline $\mathrm{FM}(\mathrm{kg})$ & .041 & .056 & .040 & .722 & .476 & -.075 & .729 & .729 & 1.371 \\
\hline
\end{tabular}

${ }^{a}$ Dependent variable FFM $(\mathrm{kg}) 24$ months

FFM fat-free mass, $F M$ fat mass, $M$ months

found also a significant and early reduction in BMR starting 1 month after the BS, although measured by BIA that was significantly correlated with FFM at all timepoints and showed no relation with the FM. These findings suggest the role of FFM in the weight homeostasis and metabolism and point out to the importance of centring the attention on the FFM and a more personalized approach of patients with obesity that are proposed for weight loss interventions. Nevertheless, at present, there is no data in large cohorts of patients on the role of the FFM in the evolution after weight loss interventions, since body composition is not usually taken into consideration in the daily clinical practice. This fact represents an important gap in the management of the patients with obesity that urgently needs to be filled.

Additionally, there is very scarce data regarding the impact of BS on the FFM evolution. Vaurs et al. [16] identified two phenotypes of FFM evolution after BS and defined as "muscle spare" $=$ FFM $<15 \%$ of TWL and "muscle loss"
$=\mathrm{FFM}>15 \%$ of TWL. In our study, we found reduction in FFT $>20 \%$ of TWL at all timepoints, reaching $25 \%$ after 24 months, independently of the BS technique (RYGB or SG) which according to present published data is considered significant muscle loss.

Besides FFM and FM parameters that are usually evaluated in body composition studies, recently, a new parameter has emerged as a more sensitive biomarker of muscle mass, in particular quality: the phase angle (PA) [27]. PA makes possible to interpret the muscle cell's capacity to transmit the electrical current produced by the BIA device according to the cell's quality. PA is an attractive index, because it is independent of body weight and was associated with poor health outcomes, quality of life, and mortality [28]. It should be noted that at present, there is very few data in the literature regarding PA normal cut-offs. Most of the studies were performed including patients in a critical state in the intensive care units or with cancer, showing levels around 
$4-5^{\circ}[28,29]$. In a study of 1967 healthy subjects from the USA, the PA of age-matched individuals was $6.96^{\circ} \pm 1.10^{\circ}$ for males and $5.97^{\circ} \pm 0.83^{\circ}$ for females [30]. A larger study published by Bosy-Westphal et al. [22], including data from 210,000 healthy German individuals, proposed the cut-off for PA of $6.01^{\circ} \pm 0.75^{\circ}$ for males and $5.59^{\circ} \pm 0.72^{\circ}$ for females, with tenth percentile values of 5.14 and 4.79 , respectively. A value below this cut-off is considered sarcopenia and low muscle mass and also quality. It should be noted that none of these studies included patients with obesity or overweight.

At present, there is no data regarding the PA in subjects with obesity and/or the impact of BS on the muscle quality evaluated by this parameter. As reflected by Table 3 from the results, in our study, we found PA levels at baseline comparable to those reported in the German study [22], although slightly lower. Nevertheless, our study was not designed to evaluate sarcopenia pre-BS and we cannot make any hypothesis on the pre-BS FFM status of our patients. Actually, at present, this point represents another important gap in the management of the patients with obesity, since there is no reliable data regarding the prevalence of sarcopenia in these subjects, especially the younger ones that are usually candidates for BS. Application of different criteria to identify sarcopenia associated to obesity may therefore currently lead to substantially and clinically unacceptable variable prevalence levels (ranged from 8 to over 50\%) due the heterogeneity in the methods that were used [6]. In order to fill this gap further, large case control studies are needed in order to dispose of reliable data. In exchange, the PA was significantly lower after the BS at all timepoints compared to baseline and significantly below the normal cut-off for both females and males proposed by Bosy-Westphal et al. [22], suggesting sarcopenia. Furthermore, PA was significantly lower in the patients that underwent BYGR, despite similar FFM with SG, suggesting that RYBG might have a greater impact on the muscle quality after the BS.

As reflected by the logistic regression analysis, the important loss of FFM after the BS was independent of the age, gender, protein metabolism, or BS techniques. The only parameters associated with the FFM at 24 months after the BS in our study were pre-BS FFM and pre-BS HOMA-IR, suggesting that pre-BS conditions have an important influence on the evolution after the BS. Previous data in the literature showed that insulin resistance (IR) has an indirect relationship with the muscle mass in humans [31]. From a mechanistic point of view, interestingly, in murine models, Ostler et al. [32] demonstrated that IR, in a context of leptin signalling impairment and inflammation (that are also present in humans with obesity), induces a significant decrease in muscle size and quality. Additionally, another study in murine models performed by Wang et al. [33] showed that IR causes muscle protein degradation. Furthermore, in the same study, treatment with rosiglitazone (a drug that decreases IR) induced only a partial recovery of the muscle mass and the authors hypothesized that maybe because it did not interfere with protein synthesis.

These previous data in murine models explain our findings in the present study. Pre-BS IR might have altered the muscle mass quantity and quality and the impact of significant rapid metabolic and body weight changes induced by the BS have led to a significant and continued muscle mass loss. Furthermore, as seen in the murine model [33], the muscle mass was not recovered after the normalization of the IR. We observed the same evolution in our study, where the FFM continued to drop out at 24 months even if the HOMA-IR normalized after the first month and remained within normal limits for Spanish population [34] during the follow-up. Our findings suggest that the mechanisms of significant muscle mass loss after BS seem independent on the BS techniques or protein metabolism parameters, like transthyretin, but are far from being elucidated. This is the first study that shows a significant FFM loss after BS maintained after 24 months, having independent predictors pre-BS FFM and IR. The main limitations of our study are the lack of a control group of subjects with normal weight and the sample size. Nevertheless, the patients represented their own control and the study was aimed to evaluate the evolution during follow-up. For this reason, we consider that the main limitations had no significant impact on the results.

These findings point to the urgent need to take into consideration changing the actual clinical guidelines for the management of patients with obesity, especially those that are candidate for BS, by incorporating body composition studies (both quantitative and qualitative) in the routinary preoperatory and follow-up evaluations. BIA is a rapid, reliable, repeatable, and non-expensive test that can be easily implemented in the daily clinical practice, as part of the evaluation of patients with obesity. This action will allow to implement more personalized approach, and design-specific physical exercises, diet, and pharmacological therapy aimed at improving the pre-BS muscle mass and insulin resistance.

\section{Conclusions}

There is a significant and early loss of FFM in patients with $\mathrm{SO}$ who undergo BS, which is not related to the parameters of protein metabolism or the surgical technique used, suggesting the existence of an independent mechanism.

Author Contribution Conceptualization, A.C.; methodology, A.C, M.C., A.V.; software, M.C., E.F.; validation, A.C. and R.S.; formal analysis, M.C., E.F., A.C.; investigation, M.C., E.F., F.P., F.F., R.V., E.C., I.H., R.B., A.Z., A.V.; resources, A.C., R.B., R.V.; data curation, M.C., E.F., A.C.; writing-original draft preparation, M.C.; 
writing-review and editing, A.C., R.S.; visualization, A.C., R.S., A.C.; supervision, A.C.; project administration, A.C.; funding acquisition: A.C. All authors have read and agreed to the published version of the manuscript.

Funding Open Access Funding provided by Universitat Autonoma de Barcelona. This study was supported by grants from the Instituto de Salud Carlos III (Fondo de Investigación Sanitaria, PI20/01806). The funders had no role in study design, data collection and analysis, decision to publish, or preparation of the manuscript.

\section{Declarations}

Conflict of Interest The authors declare no competing interests.

Open Access This article is licensed under a Creative Commons Attribution 4.0 International License, which permits use, sharing, adaptation, distribution and reproduction in any medium or format, as long as you give appropriate credit to the original author(s) and the source, provide a link to the Creative Commons licence, and indicate if changes were made. The images or other third party material in this article are included in the article's Creative Commons licence, unless indicated otherwise in a credit line to the material. If material is not included in the article's Creative Commons licence and your intended use is not permitted by statutory regulation or exceeds the permitted use, you will need to obtain permission directly from the copyright holder. To view a copy of this licence, visit http://creativecommons.org/licenses/by/4.0/.

\section{References}

1. Stensel D. Obesity and diabetes. Exerc Physiol Spec Popul. 2008;13(2):21-49.

2. Mc Farlane SI. Obesity, obstructive sleep apnea and type 2 diabetes mellitus: epidemiology and pathophysiologic insights. Sleep Med Disord Int J. 2018;2(3):52-8.

3. Kahn BB, Flier JS. On diabetes: insulin resistance Obesity and insulin resistance. J Clin Invest. 2000;106(4):473-81.

4. Son JW, Lee SS, Kim SR, Yoo SJ, Cha BY, Son HY, et al. Low muscle mass and risk of type 2 diabetes in middle-aged and older adults: findings from the KoGES. Diabetologia. 2017;60(5):865-72.

5. Janssen I, Ross R. Linking age-related changes in skeletal muscle mass and composition with metabolism and disease. J Nutr Heal Aging. 2005;9(6):408-19.

6. Johnson Stoklossa CA, Sharma AM, Forhan M, Siervo M, Padwal RS, Prado CM. Prevalence of sarcopenic obesity in adults with class II/III obesity using different diagnostic criteria. J Nutr Metab. 2017;2017.

7. Khadra D, Itani L, Tannir H, Kreidieh D, El MD, El GM. Association between sarcopenic obesity and higher risk of type 2 diabetes in adults: a systematic review and meta-analysis. World J Diabetes. 2019;10(5):311-23.

8. Cruz-Jentoft AJ, Bahat G, Bauer J, Boirie Y, Bruyère $\mathrm{O}$, Cederholm T, et al. Sarcopenia: revised European consensus on definition and diagnosis. Age Ageing. 2019;48(1):16-31.

9. Sivakumar J, Chong L, Ward S, Sutherland TR, Read M, Hii MW. Body composition changes following a very-low-calorie pre-operative diet in patients undergoing bariatric surgery. Obes Surg. 2020;30(1):119-26.

10. Cruz Jentoft AJ, Baeyens JP, Bauer JM, Boirie Y, Cederholm T, Landi F, Martin FC, Michel JP, Rolland Y, Schneider SM,
Topinková E, Maurits Vandewoude MZ. Sarcopenia: consenso europeo sobre su definición y diagnóstico. Age Ageing. 2010;39(4):412-23.

11. Pateyjohns IR, Brinkworth GD, Buckley JD, Noakes M, Clifton PM. Comparison of three bioelectrical impedance methods with DXA in overweight and obese men. Obesity. 2006;14(11):2064-70.

12. Ruiz De Eguilaz MH, Martínez De Morentín B, Pérez-Diez S, Navas-Carretero S, Martínez JA. Estudio comparativo de medidas de composición corporal por absorciometría dual de rayos X, bioimpedancia y pliegues cutańeos en mujeres. An la Real Acad Nac Farm. 2010;76(2):209-22.

13. Lee K, Shin Y, Huh J, Sung YS, Lee IS, Yoon KH, et al. Recent issues on body composition imaging for sarcopenia evaluation. Korean J Radiol. 2019;20(2):205-17.

14. Sjöström L et al. for the SOSS. Effects of bariatric surgery on mortality in Swedish obese subjects. 2007.

15. Mastino D, Robert M, Betry C, Laville M, Gouillat C, Disse E. Bariatric surgery outcomes in sarcopenic obesity. Obes Surg. 2016;26(10):2355-62.

16. Vaurs C, Diméglio C, Charras L, Anduze Y, Chalret du Rieu M, Ritz P. Determinants of changes in muscle mass after bariatric surgery. Diabetes Metab [Internet] 2015;41(5):416-421. Available from: https://doi.org/10.1016/j.diabet.2015.04.003

17. García Almeida JM, García García C, Bellido Castañeda V, Bellido GD. Nuevo enfoque de la nutrición. Valoración del estado nutricional del paciente: función y composición corporal. Nutr Hosp. 2018;35(3):1-14.

18. Kyle UG, Bosaeus I, De Lorenzo AD, Deurenberg P, Elia M, Gómez JM, et al. Bioelectrical impedance analysis - part I: review of principles and methods. Clin Nutr. 2004;23(5):1226-43.

19. Kyle UG, Bosaeus I, De Lorenzo AD, Deurenberg P, Elia M, Gómez JM, et al. Bioelectrical impedance analysis - part II: utilization in clinical practice. Clin Nutr. 2004;23(6):1430-53.

20. da Silva BR, Gonzalez MC, Cereda E, Prado CM. Exploring the potential role of phase angle as a marker of oxidative stress: a narrative review. Nutrition [Internet]. 2022;93:111493. Available from: https://doi.org/10.1016/j.nut.2021.111493

21. Riddle MC, Cefalu WT, Evans PH, Gerstein HC, Nauck MA, Oh WK, et al. Consensus report: definition and interpretation of remission in type 2 diabetes. 2021;1-7.

22. Anja BW, Danielzik S, Dörhöfer RP, Later W, Wiese S, Müller MJ. Phase angle from bioelectrical impedance analysis: population reference values by age, sex, and body mass index. J Parenter Enter Nutr. 2006;30(4):309-16.

23. Rothman KJ. BMI-related errors in the measurement of obesity. Int J Obes. 2008;32:S56-9.

24. Okorodudu DO, Jumean MF, Montori VM, Romero-Corral A, Somers VK, Erwin PJ, et al. Diagnostic performance of body mass index to identify obesity as defined by body adiposity: a systematic review and meta-analysis. Int $\mathrm{J}$ Obes [Internet] 2010;34(5):791-799. Available from: https://doi.org/10.1038/ ijo. 2010.5

25. Segal-Lieberman G, Segal P, Dicker D. Revisiting the role of bmi in the guidelines for bariatric surgery. Diabetes Care. 2016;39(August):S268-73.

26. Fidilio E, Comas M, Giribés M, Cárdenas G, Vilallonga R, Palma $\mathrm{F}$, et al. Evaluation of resting energy expenditure in subjects with severe obesity and its evolution after bariatric surgery. Obes Surg. 2021

27. Thibault R, Makhlouf AM, Mulliez A, Cristina Gonzalez M, Kekstas G, Kozjek NR, et al. Fat-free mass at admission predicts 28-day mortality in intensive care unit patients: the international prospective observational study Phase Angle Project. Intensive Care Med. 2016;42(9):1445-53. 
28. Alves FD, Souza GC, Clausell N, Biolo A. Prognostic role of phase angle in hospitalized patients with acute decompensated heart failure. Clin Nutr. 2016;35(6):1530-4.

29. Norman K, Stobäus N, Zocher D, Bosy-Westphal A, Szramek A, Scheufele R, et al. Cutoff percentiles of bioelectrical phase angle predict functionality, quality of life, and mortality in patients with cancer. Am J Clin Nutr. 2010;92(3):612-9.

30. Barbosa-Silva MCG, Barros AJD, Wang J, Heymsfield SB, Pierson RN. Bioelectrical impedance analysis: population reference values for phase angle by age and sex. Am J Clin Nutr. 2005;82(1):49-52.

31. Srikanthan P, Karlamangla AS. Relative muscle mass is inversely associated with insulin resistance and prediabetes. Findings from the Third National Health and Nutrition Examination Survey. J Clin Endocrinol Metab. 2011;96(9):2898-903.
32. Ostler JE, Maurya SK, Dials J, Roof SR, Devor ST, Ziolo MT, et al. Effects of insulin resistance on skeletal muscle growth and exercise capacity in type 2 diabetic mouse models. Am J Physiol - Endocrinol Metab. 2014;306(6):592-605.

33. Wang X, Hu Z, Hu J, Du J, Mitch WE. Insulin resistance accelerates muscle protein degradation: activation of the ubiquitin-proteasome pathway by defects in muscle cell signaling. Endocrinology. 2006;147(9):4160-8.

34. Gayoso-Diz P, Otero-González A, Rodriguez-Alvarez MX, Gude F, García F, De Francisco A, et al. Insulin resistance (HOMA-IR) cut-off values and the metabolic syndrome in a general adult population: effect of gender and age: EPIRCE cross-sectional study. BMC Endocr Disord. 2013;13(Cvd).

Publisher's Note Springer Nature remains neutral with regard to jurisdictional claims in published maps and institutional affiliations. 